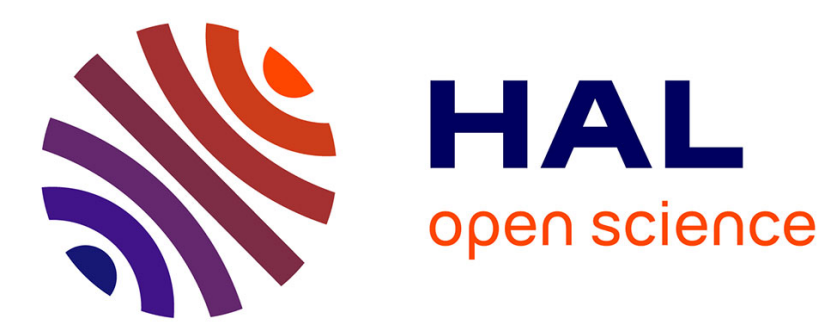

\title{
Au-delà de la productivité: les multiples mondes de la performance dans le commerce
}

Camal Gallouj, Faïz Gallouj, Marie-Hélène Vigliano

\section{To cite this version:}

Camal Gallouj, Faïz Gallouj, Marie-Hélène Vigliano. Au-delà de la productivité : les multiples mondes de la performance dans le commerce. Marché et Organisations, 2012, 15, pp.67-90. halshs-01114088

\section{HAL Id: halshs-01114088 \\ https://shs.hal.science/halshs-01114088}

Submitted on 12 Feb 2015

HAL is a multi-disciplinary open access archive for the deposit and dissemination of scientific research documents, whether they are published or not. The documents may come from teaching and research institutions in France or abroad, or from public or private research centers.
L'archive ouverte pluridisciplinaire HAL, est destinée au dépôt et à la diffusion de documents scientifiques de niveau recherche, publiés ou non, émanant des établissements d'enseignement et de recherche français ou étrangers, des laboratoires publics ou privés. 
(Publié dans la revue Marché et Organisation : GALLOUJ C., GALLOUJ F. et VIGLIANO M.-H. (2012), Réflexions sur les multiples dimensions de la performance dans le commerce et la distribution, Marché et Organisations, ${ }^{\circ} 15$, p.67-90.)

Au-delà de la productivité : les multiples mondes de la performance dans le commerce ${ }^{1}$

\author{
Camal Gallouj \\ Professeur des Universités \\ CEPN-CNRS \\ Université Paris 13 \\ IUT de Saint Denis \\ Place du 8 Mai 1945 \\ 93206 Saint Denis Cedex \\ Camal@Gallouj.com \\ 0662185877 \\ Faïz Gallouj \\ Professeur des Universités \\ CLERSE-CNRS \\ Université de Lille 1 \\ 59665 Villeneuve d'Ascq Cedex \\ Faiz.Gallouj@univ-lille1.fr
Marie-Hélène Vigliano
Maître de Conférences
Université de Paris 13
IUT de Saint Denis
Place du 8 Mai 1945 \\ 93206 Saint Denis Cedex \\ mhvigliano@gmail.com
}

\footnotetext{
${ }^{1}$ Cette communication s'appuie sur un certain nombre de travaux menés en collaboration avec Faridah Djellal.
} 


\title{
Réflexions sur les multiples dimensions de la performance dans le commerce et la distribution
}

\author{
Camal Gallouj, Faïz Gallouj et Marie-Hélène Vigliano
}

\begin{abstract}
Résumé : La question de la performance est une question récurrente dans les travaux sur le commerce et la distribution. Elle a cependant souvent été réduite (tant dans les travaux de recherche que dans les pratiques managériales) à la construction et à l'usage de ratios de productivité (selon une logique industrialiste). Nous montrons que cette manière de faire est trop restrictive et de moins en moins adaptée à un secteur commercial qui s'est fortement complexifié et dématérialisé. Nous dessinons ensuite les contours d'une approche multicritères d'évaluation de l'output commercial et nous proposons une grille de lecture des performances qui pourrait servir de base à la négociation entre acteurs.
\end{abstract}

Mots clés : productivité, performance, évaluation, services, approches multicritères

\section{Discussions on the Various Dimensions of Performance in Large Scale Retailing}

\begin{abstract}
It has nevertheless often been reduced (both in academic publications and in managerial practices) to the construct and the use of productivity ratios (following an industrialistic logic). We contend that this way of doing is rather restrictive and far from being adapted to the retailing sector which has encountered increasing complexity and intangibility. We then draw the frontiers of a multicriteria approach to evaluate the output of retailing and we build a grid which allows use to grasp the various dimensions of performance. This grid can also be used as a negotiation basis between actors and stakeholders in the field.
\end{abstract}

Key words : productivity, performance, evaluation, services, multicriteria approach 
Dans les années 80, certains travaux menés notamment aux Etats-Unis ont eu tendance à montrer que le niveau de croissance de la productivité dans le commerce et plus largement dans les services) se situait très nettement en dessous des niveaux moyens atteints par l'industrie manufacturière. Ces travaux ont contribué à conforter l'image d'un secteur commercial neutre, voire parasitaire, à faible intensité capitalistique et faible capacité d'innovation (1)

Cependant, dans le milieu des années 90, les recherches sur la performance et la productivité dans le commerce ont connu un net enrichissement, en particulier parce qu'ils ont bénéficié des développements issus de l'économie et du management des services. Ces recherches (dont les nôtres) ont montré que le commerce était un secteur singulier, caractérisé par un output relativement flou. Le commerce est en effet un service architectural de type complexe ${ }^{2}$ dont la productivité et plus généralement la performance ne peut être abordée au travers de critères simples forgés généralement pour le monde industriel.

Dans cette contribution, nous cherchons à revenir sur les difficultés d'évaluation de la performance dans le secteur du commerce. Dans une première section nous faisons rapidement état des pratiques traditionnelles d'évaluation de la performance commerciale et nous insistons tout particulièrement sur les approches en termes de productivité. Dans une deuxième section nous mettons en avant la diversité des produits commerciaux, qui fait qu'une évaluation simple de la performance est trop souvent parcellaire et restrictive. Enfin, les sections 3 et 4 sont consacrées à la mise en avant de la diversité des niveaux d'analyse de la performance dans le commerce et les relations que ces différents niveaux d'analyse peuvent entretenir entre eux.

\section{Performance commerciale et « dictature » de la productivité » : l'état du débat}

Un rapide survey des travaux sur la performance dans le secteur du commerce et de la distribution montre que c'est bien la notion de productivité et l'analyse (et la construction) de ratios de productivité qui restent au cœur des réflexions des chercheurs en management de la distribution. Cette notion de productivité continue également, selon les termes de Walters et Laffy (2), d'être une dimension centrale des préoccupations managériales de la distribution. Les données de productivité permettraient en effet de faciliter ou encore de soutenir un certain nombre de décisions tant stratégiques qu'opérationnelles. Comme le précisent Dubelaar et al. (2002, p. 417) : «Retail productivity provides vital information for number of tactical, strategic and policy related decisions in the retail industry... Clearly, given its wide use, retail productivity plays an important role in the control and management of retail organisations ». Les ratios en question sont relativement nombreux et divers. Il n'est pas nécessaire d'y revenir ici en détail. Nous en proposons néanmoins, dans l'encadré 1, une sélection parmi les plus courants et les plus diffusés auprès des professionnels et des chercheurs $(4,5,6)$.

\footnotetext{
${ }^{2}$ On aurait même assisté ces dernières années à une complexification accrue du produit dans le grand commerce en particulier (Gallouj et Gallouj, 2009).
} 


\section{Encadré 1 : Quelques exemples de ratios (« dits ») de productivité}

Espace de vente

CA au m2 (surface de vente)

Marge bénéficiaire brute par $m 2$ de surface de vente

Nombre de transactions par $m 2$ de surface de vente

Débits (nombres de passages en caisse sur une période donnée

Panier moyen (CA/débits)

Stocks

Taux de rotation des stocks

Pourcentage de marge bénéficiaire brute

Rentabilité de la marge bénéficiaire brute sur les stocks

Personnel

$C A$, ventes par employé (ventes par h. par personne)

Marge bénéficiaire brute par employé (heures travaillées)

Actif total

Ventes nettes par rapport à l'actif total

La diffusion et le succès des travaux et réflexions mais surtout l'usage courant par les managers des ratios de productivité, s'explique très largement par leur simplicité apparente. En effet, en tant que ratio ${ }^{3}$, la productivité rapporte une quantité d'output à une quantité d'input (ou ressources utilisées) contribuant ainsi à fournir des indications sur l'efficacité avec laquelle les ressources sont employées.

Derrière l'apparente simplicité de la définition de la productivité (et des ratios considérés) se cachent cependant d'énormes problèmes liés à la mesure et à la multiplicité des indicateurs possibles. Il n'est pas nécessaire de revenir sur les nombreuses difficultés techniques sousjacentes (pour plus de précisions, on se référera à l'ouvrage de Djellal et Gallouj (8) qui en propose une synthèse et une critique détaillée). Nous nous contenterons ici de relever un certain nombre de limites plus fondamentales qui font que les analyses en termes de productivité sont, dans le secteur commercial, fortement sujettes à caution.

1). Les travaux existants sur la productivité et la performance dans le champs commercial souffrent pour l'essentiel d'un des biais maintes fois soulignés par les chercheurs en économie et management des services, à savoir que les outils et concepts de mesure de la performance (de la productivité) les plus utilisés sont en fait empruntés directement aux pratiques de l'industrie. Autrement dit, ils ne sont que faiblement adaptés à l'évaluation de la performance commerciale. Nooteboom (9), parmi d'autres, nous met en garde justement contre les transferts mécaniques de notion industrielles ou industrialistes vers les activités commerciales : "(manufacturing) industry provides a utility of form, while retailing provides a utility of time and place. In view of thoses differences, one should not readily and uncritically employ concepts and tools from studies of productivity in industry $»$.

\footnotetext{
${ }^{3}$ Les ratios en question sont habituellement dérivés de quantités et pas d'unités monétaires. Dans le cas contraire on ne sait pas à priori si les améliorations éventuellement observées sont liées à une meilleure utilisation des ressources ou simplement à une fluctuation monétaire.
} 
2). La recherche de la productivité à tout prix entraine certaines externalités négatives tant à court terme qu'à long terme ${ }^{4}$. Ainsi, elle peut induire un certain nombre de coûts sociaux (stress, problèmes de santé...) ou écologiques (dégradation de l'environnement) qui ne sont pas pris en compte généralement dans l'évaluation de la productivité.

Au niveau «micro », la relation fréquemment dénoncée entre des stratégies trop agressives de productivité et la dégradation de la qualité (du service) est bien connue...

3). L'un des grands intérêts à priori des mesures de productivité réside dans leur utilisation dans une perspective de benchmarking inter et intra-organisationnel. Or, si l'on se limite au seul niveau intra-organisationnel, on peut montrer que ces mesures et comparaisons de productivité posent un problème d'équité et de justice. Elles sont souvent injustes, contreproductives et décourageantes pour les entités concernées. Ces dernières exercent en effet leurs activités dans des environnements socio-économiques (niveau de la demande locale, intensité de la concurrence locale...) souvent très différents et qui supportent difficilement les comparaisons trop mécaniques (10) ;

4). De nombreux travaux ont montré que dans les services, l'output exerce ses effets dans le temps. Il est nécessaire de distinguer le service commercial à court terme (service en actes) de ses effets à moyen et long termes. La littérature anglo-saxonne distingue ainsi l'output de l'outcome (le résultat à long terme). Les mesures de productivité ne tiennent compte que de la première dimension : le service commercial en actes ou output immédiat. Or, de plus en plus, la montée en puissance de la dimension servicielle du grand commerce contribue à mettre en avant l'importance de l'outcome ou encore de l'output médiat ;

5). Dans une économie caractérisée par des changements rapides, la composante qualitative de la performance n'est pas prise en compte par les mesures de productivité (11). En effet, de plus en plus, les distributeurs sont confrontés à une concurrence «non-prix » (par la qualité, l'amélioration du service, l'offre de services complémentaires). Autrement dit, des ressources importantes sont désormais consacrées à l'amélioration de l'offre et au développement de nouvelles prestations. Or, les mesures de productivité ne visent aucunement à prendre en considération les résultats de tels efforts et elles ne le font d'ailleurs pas. Dans un tel cadre, on comprend que le temps de travail additionnel consacré à accroître la compétitivité au travers de l'amélioration de l'offre aura pour effet mécanique une réduction de la productivité en ce sens que ces mesures prendront en considération l'usage accru des inputs mais pas les améliorations qualitatives du produit ou de la prestation qui en résultent (12).

La gestion du «secteur caisses » en grande distribution peut fournir ici un exemple typique d'amélioration du service avec effet négatif sur la productivité. En effet, en ouvrant des caisses supplémentaires pour réduire l'attente des clients, on améliore l'utilité et la satisfaction de ces clients mais dans le même temps, dans la mesure ou l'on réduit le débit ou le nombre de passages client par caisse ${ }^{5}$, on réduit de fait la productivité du secteur.

Dans des travaux remarquables comparant la grande distribution alimentaire américaine et française, Jean Gadrey (13) montre que le niveau absolu de productivité du travail est nettement plus élevé en France qu'aux Etats-Unis. La grande distribution américaine serait coupable de sous productivité chronique vis-à-vis de son homologue française et c'est ce qui expliquerait ses bonnes performances, souvent évoquées, en matière d'emplois. Comme le note ce même auteur (13) : «les performances comptables en matière de croissance et de productivité de la grande

\footnotetext{
${ }^{4}$ On pourrait avoir le même type de raisonnement en ce qui concerne le concept de croissance.

${ }^{5}$ Sauf bien entendu si cette amélioration de la qualité du service contribue à accroitre la part de marché du distributeur... ce qui est plausible mais plutôt à moyen ou long terme.
} 
distribution alimentaire américaine sont proprement lamentables si on les compare à celles de son homologue français au cours des années 80 (mais également au delà) ${ }^{6}$. Et pourtant, elle crée des emplois par millions, elle réalise des marges confortables, elle répond bien à la demande, elle est soumise à une concurrence rude, elle innove et dispose d'une réelle avance technologique. Et, lorsque les grandes entreprises françaises «hyper-productives » de la grande distribution cherchent à la concurrencer outre atlantique, elles échouent piteusement !...». Partant de ce constat, Gadrey propose ainsi d'abandonner le paradigme industriel de la productivité et d'entrer dans le paradigme d'évaluation multicritères du développement de la valeur ajoutée en service rendus. C'est ce que nous nous proposons de faire dans les sections qui suivent.

\section{La diversité et la complexification des « produits » commerciaux}

Certains travaux menés dans les années 70 et 80 ont eu tendance à considérer que le rôle des distributeurs se réduit, au travers de la conjugaison de trois fonctions de transformation de lieu (transport) de temps (stockage, entreposage) et de lots (groupage, fractionnement) à amener les produits de consommation matériellement inchangés d'un état économique à l'autre, de l'état distributif de production à l'état distributif de consommation (14). Good (15) va plus loin en considérant pour sa part que le produit commercial est essentiellement constitué d'un ensemble de services qui accroissent l'utilité du produit pour le consommateur.

Dans la réalité, il reste que le produit ou l'output commercial est extrêmement difficile à définir et à évaluer. En effet, comme la plupart des activités de service, le commerce présente de multiples spécificités qui font que les pratiques d'évaluation ne peuvent pas s'appuyer sur des critères simples. Du fait de ces spécificités, on se trouve confronté, dans le cas de la grande distribution, à une situation où, face à la multiplicité des «stakeholders », il n'y a pas d'accord des protagonistes sur les objectifs, les résultats et les moyens (contrairement à ce que l'on suppose sur un marché standard de «produits » aux caractéristiques objectives et connues de tous). Il est donc nécessaire de mettre en place des processus politiques de justification ou jugement des contributions respectives. Ces aspects conduisent à s'interroger sur les raisons qui font que l'accord n'est pas simple et qu'il ne peut s'appuyer sur des grandeurs admises par tous.

Il semble que l'on puisse apporter deux réponses à ce type de questions. L'accord n'est pas simple pour les raisons suivantes :

- d'abord et surtout en raison d'incertitudes radicales de part et d'autre sur la nature et la qualité $\mathrm{du}$ «produit fourni » ainsi parfois que sur les moyens (y compris humains) nécessaires à sa production, et sur la relation entre les fins et les moyens ;

- ensuite parce que les critères d'attribution d'une certaine valeur à ce produit peuvent diverger (par ex : procédures reconnues comme valables, résultats techniques ou résultats financiers...).

Les difficultés que nous évoquons ici sont par ailleurs amplifiées par les mutations récentes du commerce et en particulier par la complexification croissante du produit qui le caractérise. En analysant sur le long terme l'évolution de la productivité du travail dans les principaux secteurs de services, Gadrey (16) parvient à construire une courbe en $\mathrm{S}$ qui résume les transformations de l'organisation des techniques de production et de la nature des produits services correspondants. Cette courbe comprend quatre étapes distinctes. La première correspond à la phase généralement lente de modernisation du service traditionnel. Durant cette phase, les gains de productivité sont relativement faibles. La deuxième phase voie l'émergence de la production (distribution) de masse de services standardisés (et généralement peu diversifiés). Cette phase s'appuie sur des technologies centralisées qui génèrent des économies d'échelle et des gains de productivité très

\footnotetext{
${ }^{6}$ La parenthèse est de nous.
} 
importants. La phase 3 correspond à une étape ou les gains de productivité réalisés se font à un rythme inférieur. On assiste à une forte diversification des produits services, à la montée en puissance des activités marketing et de l'innovation (17) et à une vive concurrence selon la logique des «économies de variété ». Dans certains cas (cas de la distribution américaine en particulier), on atteint un dernier stade où la productivité stagne en apparence (voire régresse). En fait, selon Gadrey : «les mesures classiques de productivité deviennent de plus en plus incapables de refléter la modernisation de l'activité, fondée sur une stratégie de « services à valeur ajoutée », de solutions innovantes adaptées à des usagers multiples...».

Figure 1 : Une courbe en $\mathrm{S}$ de la productivité dans les services

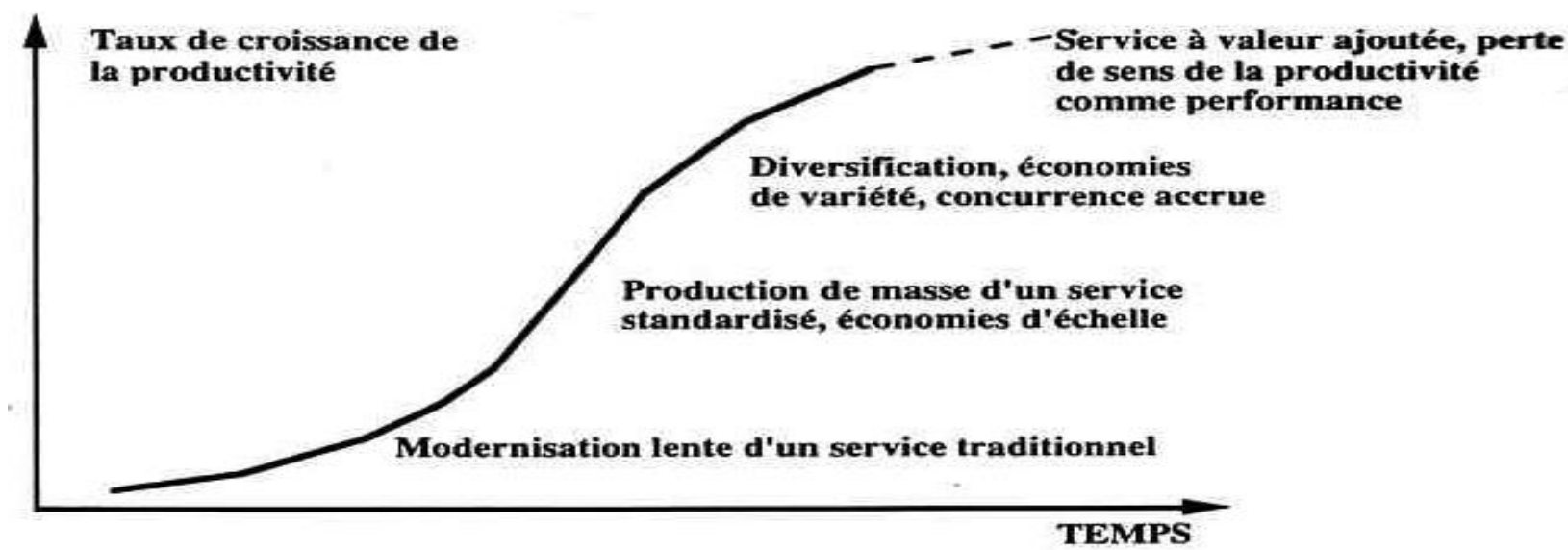

Source : Gadrey (1999)

Dans le cadre d'un précédent colloque Etienne Thil, nous avons montré, en nous appuyant sur une décomposition fonctionnelle $\mathrm{du}$ produit commercial (7), comment les dimensions informationnelles (I) puis relationnelles et servicielles (R) et cognitives (K) prennent de plus en plus le pas sur les dimensions matérielles $(\mathrm{M})$ (18). Ce phénomène illustre bien la tendance à la complexification et à la dématérialisation de la prestation commerciale.

Figure 2 : La complexification du produit dans le grand commerce

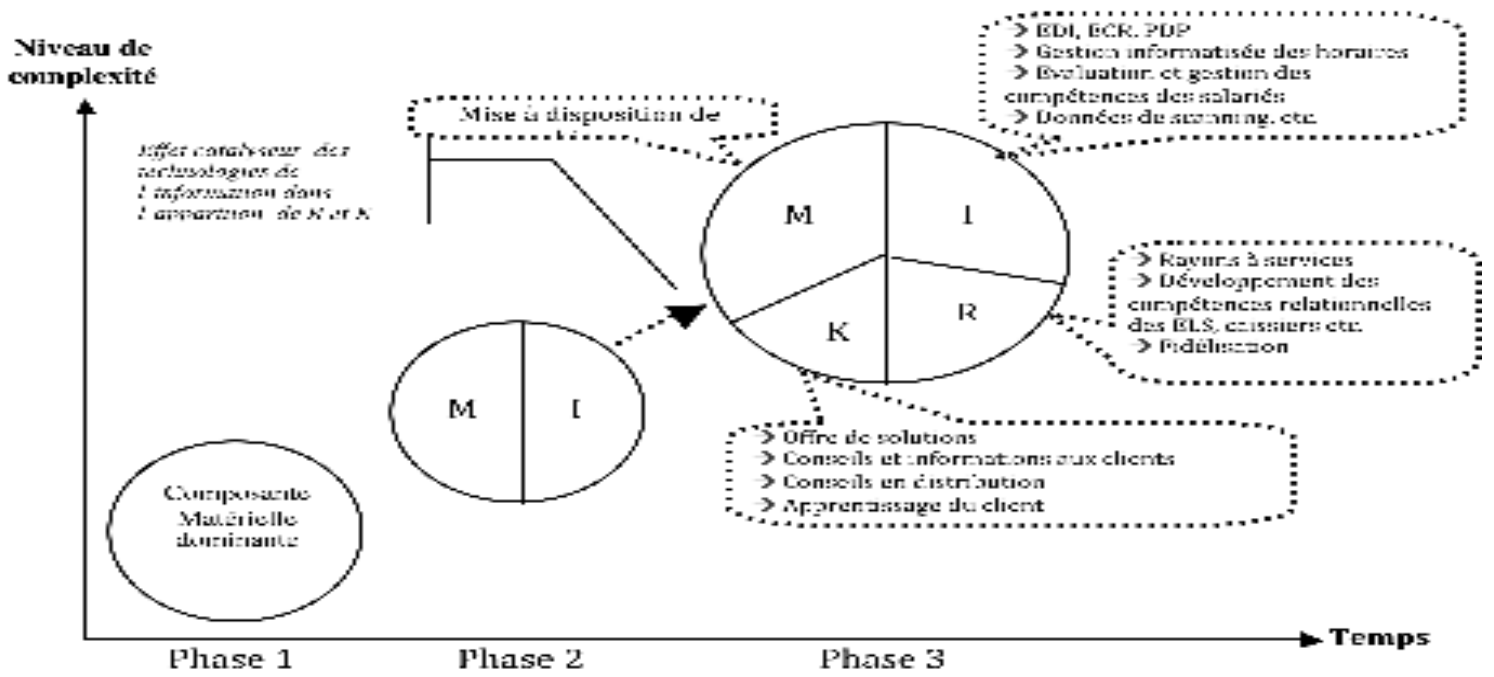

Source : Gallouj, (2007) ; Gallouj et Gallouj (2009) 
Or, cette évolution vers une forme de dématérialisation et d'accroissement du contenu en service et en connaissance du commerce (offre de solution, conseil client, etc.) contribue à diversifier et complexifier le produit commercial; autrement dit à réduire la pertinence de pratiques traditionnelles d'analyse et de mesure de la performance en termes de ratios de productivité industriels, techniques et financiers. Il apparaît dès lors nécessaire de mettre à plat les différentes catégories de services produits (ou rendus) par le commerce et les performances et/ou de critères de performance qui leur sont attachés.

Il nous semble ainsi, en nous inspirant des travaux de Boltanski et Thevenot (19) que l'on peut rendre compte de la diversité des produits commerciaux en distinguant six mondes ou familles de critères de définition et d'évaluation des «produits » et performances :

1) le monde des volumes et des opérations techniques élémentaires (monde industriel et technique) ;

2) le monde de la valeur, des opérations monétaires et financières et de la concurrence marchande (monde marchand et financier) ;

3) le monde des relations interpersonnelles de l'empathie et des liens de confiance (monde relationnel ;

4) le monde des relations sociales fondés sur le souci d'égalité de traitement, de l'équité et de la justice (monde civique) ;

5) le monde de la conception et de l'introduction de projets innovants (monde de l'innovation);

6) le monde de l'expérience, des émotions, de l'image et de la réputation.

A chacun de ces critères ou de ces mondes, on peut associer des produits génériques différents, des conceptions différentes de la qualité des produits (ou résultats) et des performances différentes. Nous les détaillons dans ce qui suit :

Des produits industriels et techniques. On fait référence à des opérations techniques élémentaires, à des volumes, des flux typiques du monde industriel. Dans le commerce, les opérations industrielles et techniques ou encore les opérations de logistique et de transformation matérielles sont très importantes et consomment encore aujourd'hui beaucoup de ressources. En effet, une large part ${ }^{7}$ de l'activité commerciale consiste à traiter des objets tangibles, des biens, c'est-à-dire à les transporter, les transformer éventuellement, les mettre à disposition, les entretenir, les réparer. On est bien ici dans une logique classique de volumes, d'actes. Les tâches concernées couvrent la construction de l'assortiment (largeur et profondeur), les relations amont (logistique et transport), la gestion des entrepôts (réception des fournisseurs et des marchandises, gestion des réserves, préparation des commandes client ou points de vente, livraison...), la gestion du linéaire (avec l'implantation des produits dans les rayons..), la gestion des flux de clients (passage en caisses...), etc.

Des produits marchands et financiers. On envisage ici le produit en termes de valeur, d'opérations monétaires et financières. La plupart des flux liés aux opérations de logistique et de transformation matérielle se doublent d'opérations financières; autrement dit de produits financiers. Les flux financiers qui traversent le commerce sont en effet énormes, qui sont liés aux dépenses clients ou aux achats et investissements du distributeur. Ces flux prennent d'ailleurs de plus en plus d'ampleur avec le phénomène bien connu de financiarisation croissante des grands groupes de distribution.

\footnotetext{
${ }^{7}$ Mais qui devient selon nous de moins en moins importante dans la création de valeur du secteur. Néanmoins, trop de travaux (principalement en économie mais également en sciences de gestion) continuent de cantonner le commerce à cet type exclusif de fonction et aux catégories d'activités qui le composent
} 
Des produits relationnels qui renvoient quant à eux au monde de l'empathie, de la relation de service, des liens de confiance. Plus précisément, ces critères font référence aux services sur mesure, aux arrangements interpersonnels rendus possibles et renforcés par la proximité, la répétition des interactions de service. Ces produits relationnels sont souvent considérés comme partie intégrante du petit commerce. Dans le grand commerce, elles ont été longtemps plus ou moins occultées par la généralisation remarquable du self service. Néanmoins, ces produits deviennent déterminants pour la plupart des enseignes, en particulier dans un souci de renforcement des liens au client et dans la perspective d'accroître sa fidélisation. Il faut noter cependant que si la répétition de ces interactions renforce la production de «produits relationnels ", ces derniers en retour produisent de la fidélité (c'est-à-dire une tendance à la répétition des interactions). De ce point de vue, on ne peut pas nier que les équipements commerciaux ont un effet structurant sur la vie d'une communauté. Cet argument vaut également pour ce qui est des produits civiques et écologiques.

Des produits civiques et écologiques (on pourrait également parler de produits sociaux et sociétaux) font référence au monde des relations sociales fondé sur un souci d'équité, de justice, d'égalité de traitement. Ces produits concernent tout à la fois les clients et les opérateurs de la distribution. Concernant les clients, on fait référence aux actions et prestations que les ELS ou les caissières (et plus généralement l'ensemble du personnel en contact) peuvent fournir aux clients en difficultés cognitive, économiques et sociales. Ces différentes prestations peuvent être envisagées comme des opérations de «réparation » de difficultés liées aux handicaps cognitifs, à l'isolement spatial et parfois affectif, aux conditions économiques et sociales... (20). Les exemples sont aujourd'hui nombreux de ces «produits » civiques, écologiques et sociétaux lancés et/ou soutenus par la grande distribution. Nous en proposons quelques illustrations dans l'encadré 2

\section{Encadré 2 : Exemples de produits « civiques » dans la grande distribution}

- Monoprix et d'autres enseignes qui s'engagent dans une démarche de santé publique en signant un pacte contre l'obésité ;

- les opérations «essentiels de la rentrée» menées sous l'égide du Ministère de l'éducation nationale, depuis 2007, et qui s'appuient sur un accord avec les enseignes nationales de la grande distribution et de la distribution spécialisée, destiné à modérer le coût de la rentrée scolaire pour les élèves. Cette opération n'est cependant pas reconduite en 2011.

- les opérations «paniers des essentiels » signées par 9 enseignes et qui visent à proposer chaque semaine à un prix préférentiel une composition comportant une dizaine de produits alimentaires de qualité...

\section{Source : divers presse}

Concernant les opérateurs, ce qui est en jeu ici, ce sont bien les questions de responsabilité de l'employeur vis-à-vis de ses salariés. Elles impliquent toute une série d'actions visant la création d'emplois, l'amélioration de la santé et la sécurité des salariés, la mise en place de conditions épanouissantes et impliquantes pour le développement personnel (motivation, formation professionnelle, employabilité...), les relations de travail (rétribution équitables et surtout lutte contre les discriminations et la gestion et la promotion de la diversité) (21), la qualité de la communication interne, la capacité d'ascension sociale au sein de l'entreprise, la participation du personnel aux résultats de l'enseigne.

Si l'on revient à la dimension écologique proprement dite, (22), on constate, que ce soit dans une logique réactive (logique opportuniste de type «markethique » ou «greenwashing »), défensive (adaptation à minima des équipements, produits et pratiques) ou offensive (volonté de créer de la valeur durable et mise en place de stratégies pionnières en la matière), que le commerce, et en 
particulier le grand commerce, s'inscrit maintenant très nettement dans des logiques de développement durable et de relations équitables avec ses fournisseurs et sous traitants. La plupart des enseignes ont depuis longtemps intégré la dimension écologique (et plus généralement sociétale) à leurs valeurs, produits et stratégies. Cette intégration passe par différents moyens (généralisation des politiques d'économie d'énergie, valorisation d'une logistique durable, engagement dans des pratiques de commerce équitable...), qu'il n'est pas utile de rappeler ici de manière exhaustive.

Des produits expérientiels, d'image et de réputation. L'utilité du consommateur n'est pas issue uniquement de la fonction approvisionnement du commerce. Elle est également et de plus en plus liée à la stimulation que cette distribution peut procurer au consommateur. La mise en scène des magasins et de leur offre constitue de plus en plus une source de positionnement dans l'environnement concurrentiel. Autrement dit, la valeur expérientielle d'un point de vente devient un paramètre important dans sa conception et dans l'analyse de sa perception par les clients (23) et de l'image qu'ils auront du magasin concerné.

Des produits d'innovation. Le grand commerce est traditionnellement considéré comme un secteur peu innovateur et, ce n'est que très rarement, que les travaux d'économie ou de gestion associent commerce et innovation ${ }^{8}$. Pour notre part, nous avons montré (18) que si le commerce apparaît au premier abord comme moins innovateur que certains autres secteurs industriels par exemple, c'est tout simplement parce que là encore, nos outils de mesure de l'innovation ne sont pas adaptés à ce type de secteur. Nous avons également montré que le grand commerce est, dans beaucoup de cas, nettement plus innovant que la plupart des secteurs industriels traditionnels. Les formes d'innovation qui sont observées dans ce secteur sont multiples et variées (nouvelles méthodes de ventes, nouveaux concepts ou formats de magasins, nouveaux produits ou services, nouveaux process ou formes d'organisation...), à la fois technologiques et non technologiques.

On distinguera ainsi comme produit de ce monde de la créativité et de l'innovation l'ensemble des solutions (éventuellement innovations incrémentales) apportées aux problèmes rencontrés lors de l'expérimentation de projets innovants, les résultats de l'apprentissage, la production de routines transférables.

\section{La diversité des performances}

Les performances doivent être envisagées en fonction des missions explicites ou implicites (contractuelles ou conventionnelles). Ainsi, comme il existe une diversité de «produits, il existe une diversité de performances. A chaque famille de «produits » génériques, on peut associer une famille de performances renvoyant à un «monde « différent. La performance est ici entendue comme l'amélioration des «positions » ou des « rendements » relatifs aux produits envisagés. On distinguera ainsi des performances industrielles et techniques, des performances marchandes et financières, des performances relationnelles, des performances civiques et écologiques, des performances de créativité et d'innovation, des performances expérientielles, d'image et de réputation (cf. tableau 1).

\footnotetext{
${ }^{8}$ Lorsque certaines études ou recherches font malgré tout, cette association, c'est généralement pour tomber dans un biais de nature technologiste. Le commerce serait utilisateur de technologies; il se contenterait dans ce cadre d'adopter des technologies (TIC dans l'essentiel des cas) produites par d'autres. En ce sens, (comme nous l'avons vu précédemment) il relèverait en matière d'innovation des secteurs dominés par les fournisseurs de technologies.
} 
L'illustration des deux premières catégories: performance industrielle et technique et performance marchande et financière ne pose généralement pas véritablement de difficultés. D'ailleurs, l'essentiel des travaux existants se caractérise par un biais fondamental en faveur de ces catégories (qui renvoient pour l'essentiel aux indicateurs classiques de productivité que nous avons abordés plus haut).

La mesure de ces types de performance soulève cependant de redoutables problèmes techniques (qui se résument en une question : que mettre au numérateur d'un ratio de performance technique ou marchande). De nombreux indicateurs existent et sont utilisés quelles que soient leurs limites. Il n'est pas nécessaire d'y revenir ici en détail ; nous en avons présenté rapidement un certain nombre dans notre section 1 . Nous nous contenterons de citer quelques exemples. Ainsi, dans le premier cas, on citera le suivi et l'analyse de l'évolution de la multitude des ratios de type trafic rapporté à des heures (du temps), des surfaces, ou par exemple les débits simples (nombre de passages en caisse). Dans le cas de la performance marchande et financière, on peut citer la progression du chiffre d'affaires (CA), le CA par salarié, le CA au m2, l'évolution du panier moyen; mais il en existe bien d'autres. Ce qui est en jeu ici c'est bien la «bonne gestion de la structure commerciale ». Notons que là aussi, les interrogations sont nombreuses quant à quels types d'indicateurs ou de ratios sont les plus pertinents pour mesurer la performance financière (24).

L'évaluation des autres types de performances (relationnelle, civique et écologique, d'innovation et expérientielle) est quant à elle nettement plus délicate et problématique.

La performance relationnelle ne consiste pas, bien entendu, à mesurer des intensités de relation, d'autant que la sociologie nous met en garde contre le caractère composite de la relation de service, envisagée comme lieu d'échange verbal d'informations techniques, d'informations marchandes et de signes de civilité et d'estime réciproque. En revanche rien n'empêche de mesurer la quantité de temps passée dans la relation ni même, après en avoir examiné les contours, la quantité de relation de chaque type. On considérera ainsi que l'amélioration des indicateurs classiques de satisfaction de la clientèle: taux de réclamation clients, taux de nourriture ${ }^{9}$, taux de fidélité ou encore taux de réduction du turnover... sont des indicateurs de performance relationnelle. Néanmoins, là encore, ces indicateurs comportent un certain nombre de limites. Ainsi par exemple, le taux de réclamation ignore de fait que la majorité des clients insatisfaits (optant plus souvent pour l'exit que pour la voice) ne font généralement pas de réclamation. De ce point de vue, les indicateurs qualitatifs et baromètres client (enquêtes consommateurs, enquêtes image) semblent nettement plus pertinents.

Lorsque l'on s'intéresse à la performance civique et écologique, il peut sembler paradoxal, par exemple, d'envisager des relations sociales ou civiques (qu'on associe généralement à des attitudes désintéressées ou de don contre don) en termes de performance (notion à connotation technique et marchande forte). L'évolution de la production et de la part des « quasi-prestations sociales » peut permettre, dans une certaine mesure, de suivre l'évolution de la performance civique. La performance écologique (ou environnementale) renvoie quant à elle à l'effort de rationalisation mené par l'entreprise de distribution en matière de consommation de ressources (énergie, consommation de matières premières, emballage), réduction des risques d'atteinte à l'environnement, valorisation des déchets...

Les questions à se poser concernant les performance civiques pourraient être : sommes nous bons, avons nous progressé en termes de contribution au lien social ; à la réduction des inégalités (accès aux produits), à la prévention et à l'éducation, à la qualité de l'environnement, à l'aménagement

\footnotetext{
${ }^{9}$ Mesure la fidélité à une marque. Il correspond à la part de marché d'une marque chez les consommateurs de cette marque.
} 
du territoire. Concernant le volet écologique, les questions à se poser pourraient être : comment a évolué le nombre de nos «filières qualité », de nos «contrats équitables ». Sommes nous bons, avons nous progressé en matière de réduction d'émissions de $\mathrm{CO} 2$ (durant le transport de marchandises), avons nous progressé en matière de tri et de recyclage des déchets...

L'innovation joue un rôle de plus en plus important dans le commerce et mobilise des quantités non négligeables de ressources et de temps. Que les instruments de gestion ne les prennent pas en compte risque d'être dommageable à long terme pour le commerce.

Le taux d'innovation (incrémentales) introduite et le taux de solution aux problèmes rencontrés lors de l'expérimentation d'un projet d'innovation ou encore le taux de ces solutions codifiées (routinisées) et transférés pour une application généralisée constituent des indicateurs de performance d'innovation.

Les questions qui sont posées ici seront les suivantes : sommes nous bons, avons nous progressé en termes de contribution à l'innovation, combien d'innovations avons nous introduites, quelles est l'intensité de ces innovations (radicales, incrémentales...).

En ce qui concerne cette fois les performances expérientielles, d'image et de réputation, on peut noter que Dupuis et Le Jean Savreux (25) figurent parmi les rares auteurs à envisager le lien entre marketing expérientiel et performance. Les auteurs en concluent qu'il est prématuré de tirer des conclusions d'enseignes expérientielles... mais que dans un certain nombre de cas, «le réenchantement d'atmosphère apparaît comme moins concluant que celui d'enseignes traditionnelles ». En réalité, la faible performance évoquée renvoie aux performances financières et économiques (de court terme). Or, là encore, ce qui doit être mesuré, c'est bien la dimension qualitative autrement dit, il s'agit d'apporter des réponses aux questions suivantes : avons nous progressé ou pas en termes de notoriété et d'image? Avons nous progressé en termes de satisfaction et de fidélisation des clients? 
Tableau 1 : Une grille multicritères d'analyse du produit et des performances dans le grand commerce

\begin{tabular}{|c|c|c|c|c|c|c|}
\hline & $\begin{array}{l}\text { Monde industriel et } \\
\text { technique }\end{array}$ & $\begin{array}{l}\text { Monde marchand et } \\
\text { financier }\end{array}$ & Monde relationnel & $\begin{array}{l}\text { Monde civique et } \\
\text { écologique }\end{array}$ & $\begin{array}{l}\text { Monde de la créativité } \\
\text { et de l'innovation }\end{array}$ & $\begin{array}{l}\text { Monde de l'expérience, } \\
\text { de l'image et de la } \\
\text { réputation }\end{array}$ \\
\hline $\begin{array}{l}\text { Produit direct } \\
\text { (output) }\end{array}$ & $\begin{array}{l}\text { Produit: } \\
\text { Ex : volume de ventes } \\
\text { Flux d'objets traités } \\
\text { Divers indicateurs de } \\
\text { trafic } \\
\text { Performance : } \\
\text { Ex; productivité } \\
\text { technique } \\
\text { Capacité de traitement } \\
\text { plus rapide des } \\
\text { opérations techniques } \\
\text { Réduction des délais et } \\
\text { des taux d'erreur } \\
\text { (passage en caisse) } \\
\text { Gains de productivité + } \\
\text { qualité industrielle } \\
\text { (délais, stocks, taux } \\
\text { d'erreur) }\end{array}$ & $\begin{array}{l}\text { Produit: } \\
\text { Ex : CA par produit } \\
\text { Valeur ajoutée, marge } \\
\text { Flux financiers } \\
\text { Performance } \\
\text { Amélioration des } \\
\text { indicateurs de santé } \\
\text { financière } \\
\text { Compétitivité par } \\
\text { rapport aux concurrents }\end{array}$ & $\begin{array}{l}\text { Produit } \\
\text { Ex : qualité des relations } \\
\text { directes personnel/client } \\
\text { (relations commerciales) } \\
\text { Réponses individualisées, } \\
\text { arrangements } \\
\text { interpersonnels } \\
\text { Confiance, conseils } \\
\text { personnalisés } \\
\text { Performance } \\
\text { Ex: amélioration des } \\
\text { indicateurs de satisfaction } \\
\text { de la clientèle à l'égard de } \\
\text { la relation } \\
\text { Amélioration des qualités } \\
\text { relationnelles du servie }\end{array}$ & $\begin{array}{l}\text { Produit } \\
\text { Ex: Traitement } \\
\text { équitable des personnes } \\
\text { (clients, fournisseurs, } \\
\text { personnel) } \\
\text { Assistance aux } \\
\text { populations défavorisées } \\
\text { Accessibilité équitable } \\
\\
\text { Performance } \\
\text { Amélioration des } \\
\text { qualités civiques du } \\
\text { service } \\
\text { Amélioration des } \\
\text { qualités écologiques }\end{array}$ & 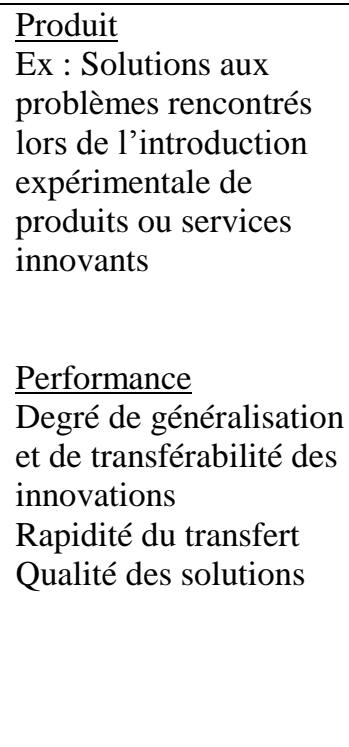 & $\begin{array}{l}\text { Produit } \\
\text { Ex : Emotion, ré- } \\
\text { enchantement } \\
\text { Image et réputation à } \\
\text { CT } \\
\text { Performance } \\
\text { Amélioration de } \\
\text { l'image et de la } \\
\text { réputation }\end{array}$ \\
\hline $\begin{array}{l}\text { Produit } \\
\text { indirect } \\
\text { (outcome) }\end{array}$ & Concept peu pertinent & $\begin{array}{l}\text { Produit } \\
\text { Stabilité et sécurité } \\
\text { financière de } \\
\text { l'entreprise (mais aussi } \\
\text { des clients) } \\
\text { Performance } \\
\text { Indicateurs de } \\
\text { rendements financiers à } \\
\text { long terme } \\
\text { Maitrise des conditions } \\
\text { financières du } \\
\text { développement à LT }\end{array}$ & $\begin{array}{l}\text { Produit } \\
\text { Fidélisation de la clientèle } \\
\text { Production de } \\
\text { comportement de fidélité } \\
\text { (à l'entreprise) ou aux } \\
\text { agents et confiance } \\
\text { réciproque } \\
\text { Performance } \\
\text { Réduction du turnover des } \\
\text { clients } \\
\text { Insertion durable de } \\
\text { l'organisation dans les } \\
\text { réseaux locaux }\end{array}$ & 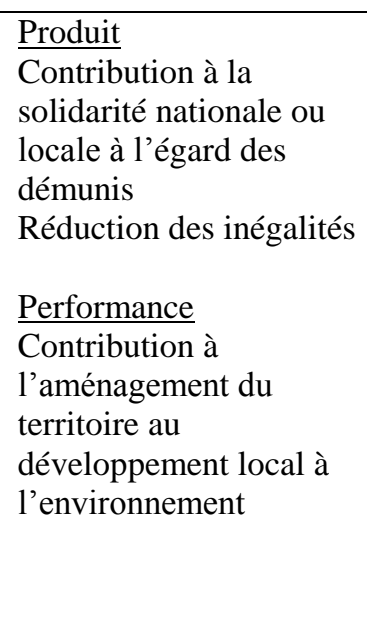 & $\begin{array}{l}\text { Produit } \\
\text { Intégration dans les } \\
\text { systèmes locaux } \\
\text { d'innovation } \\
\text { Performance } \\
\text { Amélioration du taux } \\
\text { de solution aux } \\
\text { problèmes par effet } \\
\text { d'expérience et } \\
\text { d'apprentissage } \\
\text { Amélioration de la } \\
\text { qualité des solutions } \\
\text { trouvées sur le LT } \\
\text { Amélioration de la } \\
\text { rapidité du transfert }\end{array}$ & $\begin{array}{l}\text { Performance } \\
\text { Réduction durable du } \\
\text { turnover des clients } \\
\text { Fidélisation, } \\
\text { attachement durable à } \\
\text { l'enseigne }\end{array}$ \\
\hline
\end{tabular}




\section{Les relations entre les différents niveaux de performance}

Les six catégories de performance ainsi envisagées ne sont pas exclusives bien au contraire. Elles entretiennent ou peuvent entretenir des liens entre elles, qui peuvent être à la fois positifs ou négatifs. Autrement dit, on peut parfois parler de complémentarité et de renforcement, parfois d'opposition.

Figure 3 : les différents niveaux de performance et leurs multiples interrelations

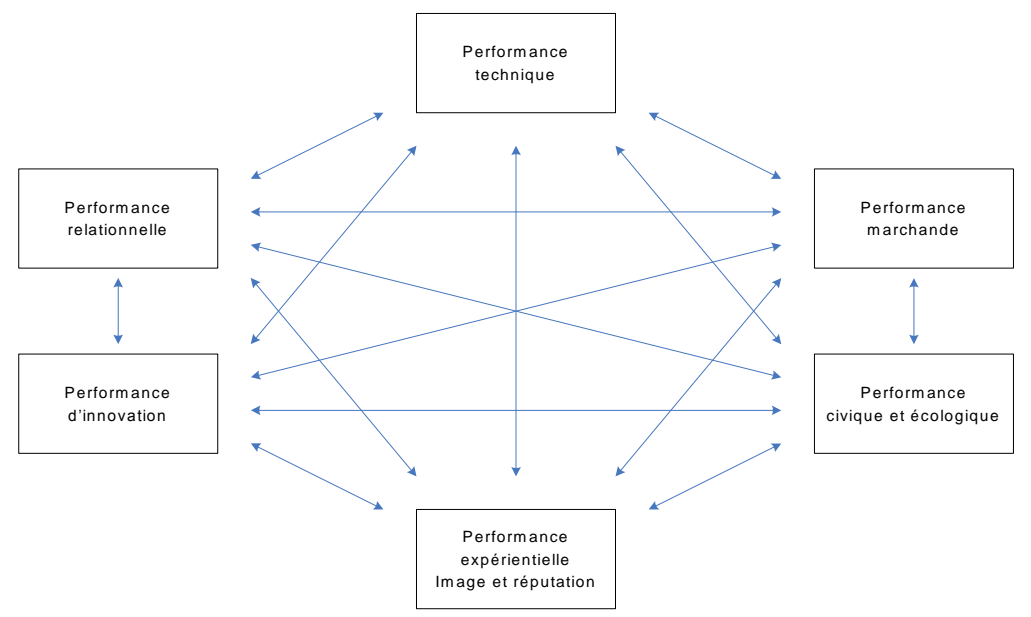

Une amélioration de la performance technique induit généralement une amélioration de la performance marchande ; cependant, l'effet inverse est également possible et cette amélioration de la performance technique peut, dans certaines conditions, contribuer à une diminution de la performance marchande (effet volume). Une amélioration de la performance relationnelle (augmentation du taux de fidélisation des clients) peut quant à elle exercer une influence positive sur la performance marchande. On peut également penser qu'une bonne performance relationnelle est susceptible d'avoir un impact très positif sur la performance innovation. En effet $(18,26)$, l'interface client et sa bonne gouvernance est souvent source de nombreuses innovations à la fois incrémentales et radicales.

La performance civique et écologique peut quant à elle se trouver en contradiction avec la performance technique (productivité) et financière. Ainsi par exemple de bonnes conditions d'emploi ou tout simplement la stabilité de l'emploi peuvent conduire à une réduction de la flexibilité ou de l'agilité des enseignes (27) par rapport aux fluctuations d'activité et des ventes inhérentes au secteur. A l'inverse, on peut également considérer (en vertu de la logique du don contre don appliquée au marché du travail) qu'un personnel stable peut se caractériser par une motivation et une efficacité supérieure, contribuant ainsi à renforcer les performances techniques et financières.

La logique expérientielle, elle aussi peut aller dans le sens d'un renforcement de la performance innovation. En effet, dans la mesure où les magasins d'expérience sont aussi souvent des magasins pédagogiques, ils contribuent à améliorer les compétences du client et à accroître sa propension à devenir partenaire de l'innovation. Cependant, la performance expérientielle et la théatralisation peuvent également avoir des effets négatifs (tout au moins à court terme) sur la performance financière. En effet, comme le précisent Dioux et Dupuis (5), le besoin de changement ressenti par les consommateurs ... pose un problème aux managers de la distribution quant à l'horizon de temps à prendre en compte dans les démarches d'investissement». 
Autrement dit, la durée de vie des concepts d'enseignes se réduit et certaines formules peuvent devenir caduques rapidement. A l'inverse, ces évolutions devraient inciter les concepteurs à réfléchir à la généralisation de la modularité des concepts et structures.

Dans certaines situations, les performances industrielle et techniques et les performances écologiques peuvent être corrélées positivement. Ainsi par exemple, on sait qu'aujourd'hui la majorité du transport de marchandises s'effectue en France par la route (plus de $80 \%$ ) Or, selon l'association ECR, plus de 8 camions sur 10 ne sont pas complets et par ailleurs la moyenne des palettes par camion qui peut théoriquement être de 33 n'est à ce jour que de 17. Ainsi, une amélioration du taux de remplissage contribue tout à la fois à l'amélioration des performances industrielles et des performances écologiques.

\section{Conclusion}

Le commerce produit de la mise à disposition de biens, mais il produit également bien d'autres choses, qu'on ne peut laisser de côté (d'autant plus qu'elles mobilisent des ressources souvent importantes). La grille que nous proposons permet selon nous de mieux tenir compte de la spécificité du système commercial et en particulier de ses caractéristiques de service de nature architecturale. Elle permet ainsi d'évaluer la performance commerciale sur plusieurs dimensions et pas seulement sur celle de sa performance technique. Elle permet également de mieux tenir compte de l'usager et de son point de vue. D'un point de vue managérial son usage est double : elle peut servir dans le cadre de négociations entre chaque niveau d'autorité d'une organisation commerciale (secteur, catégorie, entreprise, enseigne, direction régionale...); elle peut également être au cœur des comparaisons plus équitables et plus générales entre établissements, voire pays. 


\section{Références bibliographiques}

(1) Gallouj C., Vigliano M.H. (2011), Images et figures du grand commerce dans les recherches en SHS, Marchés et organisation, A paraître.

(2) Walters D., Laffy D. (1996), Managing Retail Productivity and Profitability, London, MacMillan

(3) Dubelaar C., Bhargava M., Ferrarin D. (2002), Measuring Retail Productivity : What Really Matters ?, Journal of Business Research, 55, 417-426.

(4) Anon Higon D. et al. (2009), The Determinants of Retail Productivity : a Critical Review of the Evidence, International Journal of Management Reviews, 201-217.

(5) Dioux J., Dupuis M. (2009), La distribution: stratégie des réseaux et management d'enseignes, Pearson Education.

(6) McGoldrick P. J. (2002), Retail Marketing, Mc Graw Hill.

(7) Gallouj C., Gallouj S. (2009), L'innovation dans la grande distribution : essai de construction d'une approche servicielle, Management et Avenir, 103-120.

(8) Djellal F., Gallouj F. (2008), Measuring and Improving Productivity in Services, Edward Elgar

(9) Nooteboom B. (1986), Costs, Margin and Competition : Causes of Structural Change, in ESOMAR (eds) Retail stratégies for Profits and Growth, Amsterdam, 186-98

(10) Achabal D. D., Heineke J.M., McIntyre S.H., Issues and Perspectives on Retail Productivity, Journal of Retailing, 60, 107-127.

(11) Ratchford B.T. (2003), Has the Productivity of Retail Stores Really Declined ? Journal of Retailing, 79, 171-182.

(12) Noyelle T., Stanback T. (1991), La productivité dans les services : une mesure valable des performances économiques? 7-108, dans De Bandt ed., les services, productivité et prix, Economica.

(13) Gadrey J. (1996), Services : la productivité en question, Desclée de Brouwer

(14) Wickham S. (1976), Vers une société de consommateurs, PUF.

(15) Good W. S. (1984), Productivity in the Retail Grocery Trade, Journal of Retailing, 60, 3, 8197

(16) Gadrey J. (1999), La productivité n'est pas la performance, dans Gadrey, Gallouj et al., Comment mesurer la performance, Collection de la Mission de la Recherche de la Poste, Cahier $\mathrm{n}^{\circ} 5,3-6$

(17) Moati P., Volle P. (2011) La montée en puissance des compétences marketing dans la grande distribution, Management et Avenir, A paraître.

(18) Gallouj C. (2007), Innover dans la grande distribution, De Boeck

(19) Boltansky L., Thevenot L., (1991), De la justification : les économies de la grandeur, Paris, Gallimard.

(20) Barth N. Antéblian B. (2010), Seniors, grande distribution et courses ordinaires, Gérontologie et Société, 135, 83-114

(21) Fache P., Laude L., Waelli M. (2010), Travailler dans les services : les seniors en première ligne ; le cas des greeters de WalMart, Gérontologie et Société, 135, 115-131.

(22) Carroll A. (1979), A Three Dimensional Conceptual Model of Corporate Social Performance, Academy of Management Review, 4, 4, 497-505.

(23) Filser M., Des Garets V., Paché G. (2001), La distribution : organisation et stratégie, EMS Editions

(24) Dawson J. (2005), Output considerations in Retail Productivity, International Review of Retail, Distribution and Consumer Research, 14,3, 337-349.

(25) Dupuis M., Le Jean Savreux D. (2004), Marketing expérientiel et performance des enseignes de distribution, Revue française de marketing, 198, 89-106.

(26) Djellal F. et al. (2004), L'hôpital innovateur, Masson.

(27) Badot O. (1997), Théorie de l'entreprise agile, L'Harmattan. 